\title{
Comparison of Newton Raphson and Hard Darcy methods for gravity main nonlinear water network
}

\author{
AbdulhamidSaad, HasanAhweedi, HusamEL-DinAbdulkaleq, \\ AlwaleedKhaleel, Ahmed Abdulsalam. \\ ${ }^{a}$ Faculty of Engineering, Tobruk University, Tobruk, Libya.
}

\begin{abstract}
A water network of 24 pipes depending on mainly gravity and covers an area of 3.78 square kilometers was taken an as a case study to test and compare the analysis. The governing equation of this network are internal flow in pipe equations, which consist of the continuity equation, the modified Bernoulli's equation, and the head loss due to the length of the pipe. The three equations are nonlinear algebraic equations because of the square power of the discharge in the head loss equations, which need to be solved numerically. Hard Darcy method and Newton Raphson method are used to solve the system of nonlinear equations, and to compare the solution.So, twenty four nonlinear equations (nine Bernoulli's equations and fifteen continuity equations) in twenty four unknowns discharges were got by these two method by using MATLAB code. There are not differences in the resulted discharges between Hard Darcy and Newton Raphson methods. Also, it was found that Newton Raphson was faster than Hard Darcy Method when they compared by the number of iteration. The final solution of the discharges have tested by the basic of fluid mechanics that says the summation of head losses inside a loop must be equal zero which can be seen clearly in the plots of the two methods.
\end{abstract}

Keywords: comparison, duscharge, pipe, HardDrcy, Newton Raphson

\section{Introduction}

Water pipe network systems are designed and operated to supply fresh water from the source (or treatment facility) to customers (Hund-Der \& Yu-Chang, 2008). Nearly $80 \%$ to $85 \%$ of the cost of a total water supply system is contributed toward water transmission and the water distribution network (Abdulhamid, 2016). In this project, the distribution network of 24 pipes with nine looped network and gravity main is considered.

Analysis will take place bysetting up a system of a nonlinear equation as results of internal flow in pipe such as, the continuityequation, Bernoulli equation, and the major losses equations. This system cannot be solved analytically. Therefore, numerical method by using MATLAB software is used to solve the nonlinear systems of the network.

Nonlinear equations set can be formulated to describe the relationship between the nodal head and pipe flow rate. Hard Darcy method and Newton Raphson method was commonly used to solve the nonlinear equation set for obtaining the solution of the network (Hund-Der \& Yu-Chang,2008).

The hydraulic and optimization analysis are linked through an iterative procedure. The analysis of the pipe network is to estimate the discharge in each pipe, velocities, and the total cost of the system. Also, proof of the solution in each method and the comparison between the two will be considered.

\subsection{The modified Bernoulli equation}

The Bernoulli equation is a relation between pressure, velocity,and elevation in steady, incompressible flow(Yunus A \& John M,2006) as shown in the next equation.

$$
\frac{\mathrm{P}_{1}}{\rho}+\frac{V_{1}^{2}}{2}+g z_{1}=\frac{\mathrm{P}_{2}}{\rho}+\frac{V_{2}^{2}}{2}+g z_{2}+h_{L}
$$

Where $\frac{\mathrm{P}}{\rho}$ is the flow energy, $\frac{V^{2}}{2}$ is kinetic energy, $g z$ is potential energy and $h L$ is head losses.

\subsection{The major losses in pipe}

The head loss due to viscous effects in the straight pipes, termed the major loss and denoted $h_{\text {Lmajor }}$ (Munson et al., 2009).

$$
h_{L}=f \frac{L}{D} \frac{V^{2}}{2 g}
$$




\subsection{The minor losses in pipe}

The fluid in a typical piping system passes through various fittings, valves, bends, elbows, tees, inlets, exits, enlargements, and contractions in additionto the pipes. These components interrupt the smooth flow of the fluid andcause additional losses because of the flow separation and mixing theyinduce. In a typical system with long pipes, these losses are minor comparedto the total head loss in the pipes (the major losses) and are called minor losses (Yunus A \& John M,2006).

$h_{L_{\text {min } o r}}=K L \frac{V^{2}}{2 g}$

\subsection{Volumetric flow rate (discharge)}

The volume of the fluid flowing through a cross section per unit time is:

$Q=V A_{C}$

\subsection{Series and parallel network}

For pipes in series, the flow rate is the same in each pipe, and the total head loss is th. losses in individual pipes.

$h_{L T}=h_{L 1}+h_{L 2}+h_{L 3}$

Since the same discharge passes through all the pipes, the continuityequation is

$Q=Q_{1}=Q_{2}=Q_{3}=\ldots Q_{n}$

For pipes in parallel, the head loss is the same in each pipe, and the total fiuw rate is une sum of the flow rates in individual pipes.

$Q_{A}=Q_{1}+Q_{2}=Q_{B}$

$h_{L 1}=h_{L 2}$

\section{The Problem}

Water supply networks consistof a of sources, pipe loops (M. Tabesh,2001) in this case study design a water network from node No1 which is the upstream to node No 13 which is the downstream by gravity main as shown figure (1). The dimensions of the network are listed inTables 1and2. The network covers an area of 3.78 kilometers square, consisted of nine loops (24 pipes, main lines and minor lines) what's more, the outside border of the network considered as the main lines, and the inner lines considered as minor line of the network.

Furthermore, this network included of 16 nodes, the first node considered the upstream (with neglectedminor losses)

(Swamee\&Sharma,2008)

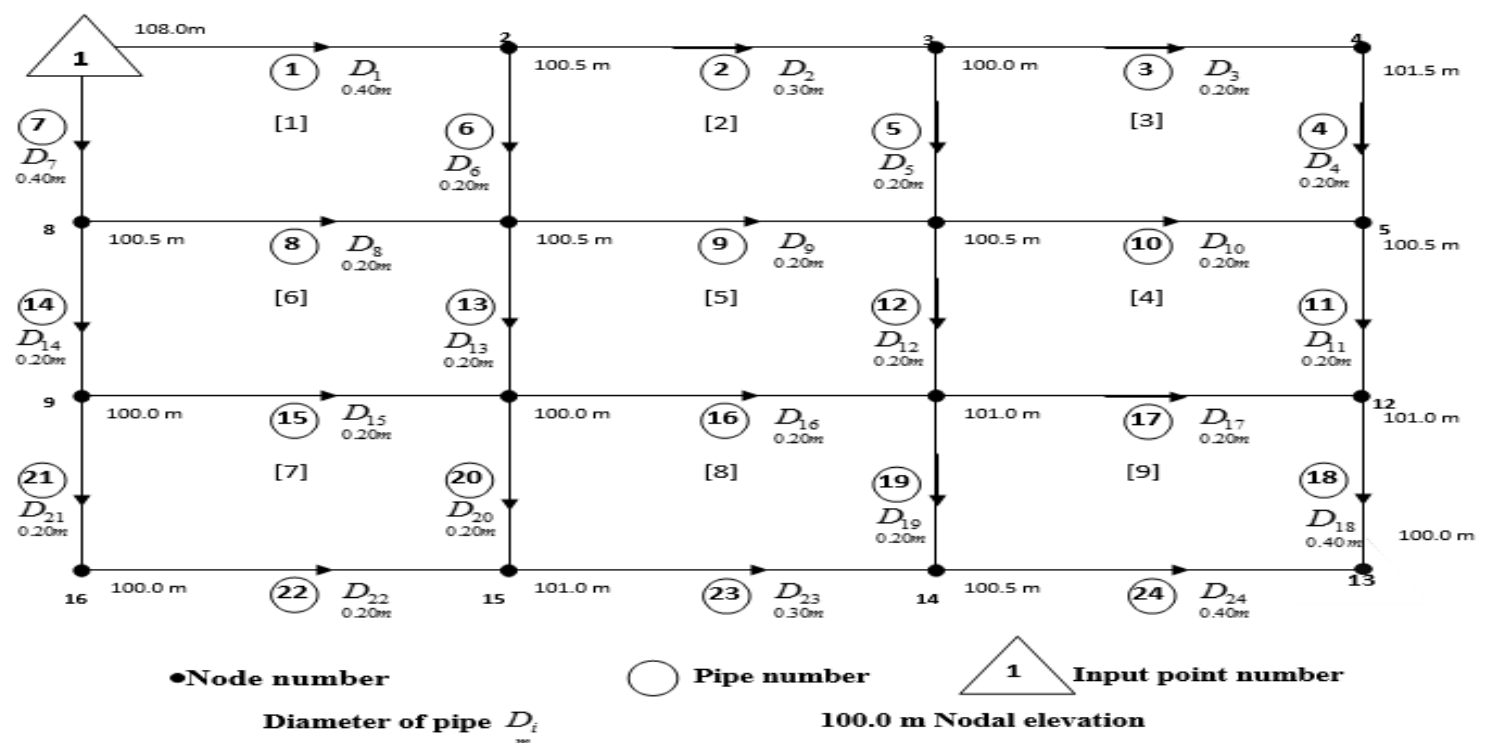

Fig 1: Gravity main looped network of 24 pipes 
Table 1: The dimensions of the network

\begin{tabular}{|l|l|l|l|l|l|l|l|l|}
\hline No. of pipe & 1 & 2 & 3 & 4 & 5 & 6 & 7 \\
\hline Diameter (m) & 0.4 & 0.3 & 0.2 & 0.2 & 0.2 & 0.2 & 0.4 \\
\hline Length (m) & 800 & 800 & 800 & 800 & 600 & 600 & 0.2 \\
\hline No. of pipe & 9 & 10 & 11 & 12 & 13 & 14 & 600 \\
\hline Diameter (m) & 0.2 & 0.2 & 0.2 & 0.2 & 0.2 & 0.2 & 600 \\
\hline Length (m) & 600 & 600 & 600 & 600 & 600 & 600 & 0.2 & 600 \\
\hline No. of pipe & 17 & 18 & 19 & 20 & 21 & 22 & 6.2 & 23 \\
\hline Diameter (m) & 0.2 & 0.4 & 0.2 & 0.2 & 0.2 & 0.2 & 0.3 & 0.4 \\
\hline Length (m) & 600 & 600 & 600 & 600 & 600 & 600 & 600 \\
\hline
\end{tabular}

Table 2: The elevation of each node

\begin{tabular}{|l|l|l|l|l|l|l|l|l|}
\hline No. of node & 1 & 2 & 3 & 4 & 5 & 6 & 7 & 8 \\
\hline Height $(\mathrm{m})$ & 108 & 100.5 & 101 & 100.5 & 100.5 & 100.5 & 100.5 & 100.5 \\
\hline No. of node & 9 & 10 & 11 & 12 & 13 & 14 & 15 & 16 \\
\hline Height $(\mathrm{m})$ & 100 & 100 & 101 & 101 & 100 & 100.5 & 101 & 100 \\
\hline
\end{tabular}

\section{Numerical Solution Of Nonlinear System Of Equations}

One of the most common important steps in water resources engineering is pipe network analysis, the key methods for this analysis are Hard Darcy and Newton-Raphson (I.A. Oke;2007).

\subsection{The assumption}

\section{The Solution By Using Newton Raphson}

All the discharges can be assumed for one value or different values as shown in table 3(Moosavian\& Jaefarzadeh, 2014).. Therefore, in Newton Raphson not necessary to assume an initial guesses that satisfies the continuity equations as shown in table 5.1.

Table 3: initial guesses of Newton Raphson method

\begin{tabular}{|l|c|c|c|c|c|c|c|c|}
\hline Pipe discharges & $Q_{1}$ & $Q_{2}$ & $Q_{3}$ & $Q_{4}$ & $Q_{5}$ & $Q_{6}$ & $Q_{7}$ & $Q_{8}$ \\
\hline The assumed values & 0.05 & 0.05 & 0.05 & 0.05 & 0.05 & 0.05 & 0.05 & 0.05 \\
\hline Pipe discharges & $Q_{9}$ & $Q_{10}$ & $Q_{11}$ & $Q_{12}$ & $Q_{13}$ & $Q_{14}$ & $Q_{15}$ & $Q_{16}$ \\
\hline The assumed values & 0.05 & 0.05 & 0.05 & 0.05 & 0.05 & 0.05 & 0.05 & 0.05 \\
\hline Pipe discharges & $Q_{17}$ & $Q_{18}$ & $Q_{19}$ & $Q_{20}$ & $Q_{21}$ & $Q_{22}$ & $Q_{23}$ & $Q_{24}$ \\
\hline The assumed values & 0.05 & 0.05 & 0.05 & 0.05 & 0.05 & 0.05 & 0.05 & 0.05 \\
\hline
\end{tabular}

4.2 The equations of Newton Raphson method

* The discharge equation of each node

We know the summation of inflow and out flow at node should be equal zero, therefore:

$F_{1}=Q_{1}+Q_{7}-0.15$

$F_{2}=Q_{4}-Q_{3}$

$F_{3}=Q_{22}-Q_{21}$

$F_{4}=Q_{2}+Q_{6}-Q_{1}$

$F_{5}=Q_{3}+Q_{5}-Q_{2}$

$F_{6}=Q_{8}+Q_{14}-Q_{7}$

$F_{7}=Q_{15}+Q_{21}-Q_{14}$

$F_{8}=Q_{9}+Q_{13}-Q_{6}-Q_{8}$

$F_{9}=Q_{16}+Q_{20}-Q_{15}-Q_{13}$

$F_{10}=Q_{18}-Q_{17}-Q_{11}$

$F_{11}=Q_{10}+Q_{12}-Q_{5}-Q_{9}$

$F_{12}=Q_{23}-Q_{22}-Q_{20}$ 


$$
\begin{aligned}
& F_{13}=Q_{19}+Q_{17}-Q_{12}-Q_{16} \\
& F_{14}=Q_{24}-Q_{19}-Q_{23} \\
& F_{15}=Q_{11}-Q_{10}-Q_{4}
\end{aligned}
$$

\section{* The head losses equation of each loop}

By using the basic of fluid mechanics, the sum of losses inside each loop should be equal zero, therefore:

$$
\begin{aligned}
& F_{16}=h_{L 1}+h_{L 6}-h_{L 8}-h_{L 7} \\
& F_{17}=h_{L 2}+h_{L 5}-h_{L 9}-h_{L 6} \\
& F_{18}=h_{L 3}+h_{L 4}-h_{L 10}-h_{L 5} \\
& F_{19}=h_{L 8}+h_{L 13}-h_{L 15}-h_{L 14} \\
& F_{20}=h_{L 9}+h_{L 12}-h_{L 13}-h_{L 16} \\
& F_{21}=h_{L 10}+h_{L 11}-h_{L 17}-h_{L 12} \\
& F_{22}=h_{L 15}+h_{L 20}-h_{L 22}-h_{L 21} \quad F_{23}=h_{L 16}+h_{L 19}-h_{L 23}-h_{L 20} \\
& F_{24}=h_{L 17}+h_{L 18}-h_{L 24}-h_{L 19} \\
& \text { Where } h_{L}=\frac{8 f L Q^{2}}{\pi^{2} g D^{5}}
\end{aligned}
$$

\subsection{Finding the jacobian}

The equation (34) has the jacobian matrix which can be found as follow.

$\left[\left(\frac{\partial f_{24 * 24}}{\partial Q}\right]_{\left(Q_{1}, Q_{2}, Q_{3}, \ldots \ldots . Q_{24}\right)}^{-1}\left[\begin{array}{cccc}\frac{\partial f_{1}}{\partial Q_{1}} & \frac{\partial f_{1}}{\partial Q_{2}} \ldots \ldots \ldots . . & \frac{\partial f_{1}}{\partial Q_{24}} \\ \vdots & & \vdots & \vdots \\ \frac{\partial f_{24}}{\partial Q_{1}} & \frac{\partial f_{24}}{\partial Q_{2}} \ldots \ldots \ldots . . & \frac{\partial f_{24}}{\partial Q_{24}}\end{array}\right]\right.$
$J^{-1}(1,1)=\frac{\partial f_{1}}{\partial Q_{1}}=\frac{\partial\left(Q_{1}+Q_{7}-0.6\right)}{\partial Q_{1}}=1$

$$
J^{-1}(24,24)=\frac{\partial f_{24}}{\partial Q_{24}}=\frac{\partial\left(h_{L 17}+h_{L 18}-h_{L 24}-h_{L 19}\right)}{\partial Q_{24}}=-56
$$

\subsection{The final matrix}

The next matrix shows the calculation of the first iteration of each loop
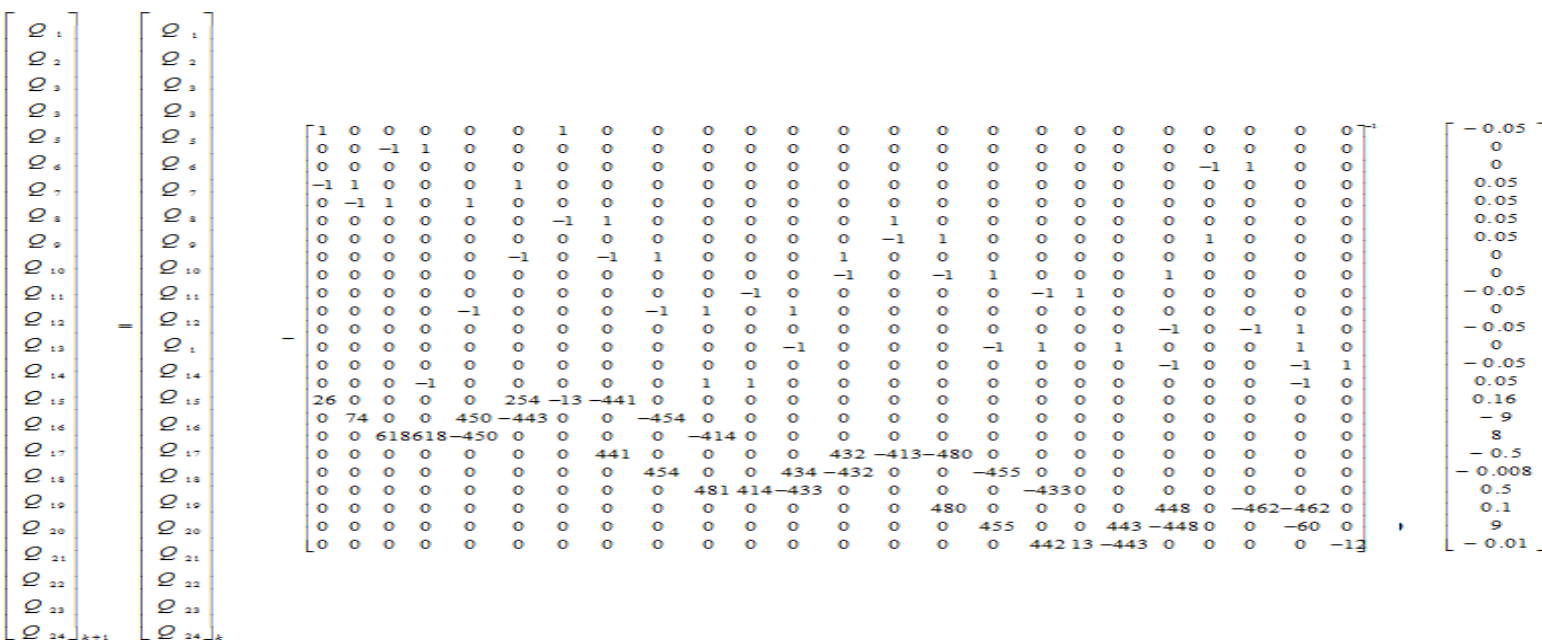


\subsection{The result of the pipe discharges of the first iteration}

Table4: The pipe discharge for the first iteration

\begin{tabular}{|l|c|c|c|c|c|c|c|c|}
\hline Pipe discharge & $Q_{1}$ & $Q_{2}$ & $Q_{3}$ & $Q_{4}$ & $Q_{5}$ & $Q_{6}$ & $Q_{7}$ & $Q_{8}$ \\
\hline Values & 0.0787 & 0.0510 & 0.0212 & 0.0212 & 0.0299 & 0.0277 & 0.0713 & 0.0293 \\
\hline Discharges & $Q_{9}$ & $Q_{10}$ & $Q_{11}$ & $Q_{12}$ & $Q_{13}$ & $Q_{14}$ & $Q_{15}$ & $Q_{16}$ \\
\hline Values & 0.0227 & 0.0189 & 0.0401 & 0.0336 & 0.0343 & 0.0420 & 0.0176 & 0.0219 \\
\hline Pipe discharge & $Q_{17}$ & $Q_{18}$ & $Q_{19}$ & $Q_{20}$ & $Q_{21}$ & $Q_{22}$ & $Q_{23}$ & $Q_{24}$ \\
\hline Values & 0.0283 & 0.0683 & 0.0273 & 0.0300 & 0.0244 & 0.0244 & 0.0544 & 0.0817 \\
\hline
\end{tabular}

\subsection{The pipe discharges and velocities of the last iteration}

The correct discharges and velocities can be got after 11 iteration (MATLAB code by using Newton Raphson method see App A), showed in table 5 and 6.

Table 5: The pipe dischargesfor the last iteration

\begin{tabular}{|l|l|l|l|l|l|l|l|l|}
\hline Pipe discharges & $Q_{1}$ & $Q_{2}$ & $Q_{3}$ & $Q_{4}$ & $Q_{5}$ & $Q_{6}$ & $Q_{7}$ & $Q_{8}$ \\
\hline Values & 0.0786 & 0.0510 & 0.0212 & 0.0212 & 0.0298 & 0.0277 & 0.0714 & 0.0293 \\
\hline Pipe discharges & $Q_{9}$ & $Q_{10}$ & $Q_{11}$ & $Q_{12}$ & $Q_{13}$ & $Q_{14}$ & $Q_{15}$ & $Q_{16}$ \\
\hline Values & 0.0227 & 0.0189 & 0.0401 & 0.0337 & 0.0343 & 0.0420 & 0.0176 & 0.0218 \\
\hline Pipe discharges & $Q_{17}$ & $Q_{18}$ & $Q_{19}$ & $Q_{20}$ & $Q_{21}$ & $Q_{22}$ & $Q_{23}$ & $Q_{24}$ \\
\hline Values & 0.0283 & 0.0683 & 0.0272 & 0.0300 & 0.0245 & 0.0245 & 0.0544 & 0.0817 \\
\hline
\end{tabular}

In addition, by apply the equation [4] $Q=v A_{C}$ we get the following velocities:

Table 6: The pipe velocitiesfor the last iteration

\begin{tabular}{|l|l|l|l|l|l|l|l|l|}
\hline Pipe velocities & $v_{1}$ & $v_{2}$ & $v_{3}$ & $v_{4}$ & $v_{5}$ & $v_{6}$ & $v_{7}$ & $v_{8}$ \\
\hline Values & 0.6259 & 0.7213 & 0.6739 & 0.6739 & 0.9491 & 0.8805 & 0.5678 & 0.9334 \\
\hline Pipe velocities & $v_{9}$ & $v_{10}$ & $v_{11}$ & $v_{12}$ & $v_{13}$ & $v_{14}$ & $v_{15}$ & $v_{16}$ \\
\hline Values & 0.7232 & 0.6012 & 1.2751 & 1.0711 & 1.0906 & 1.3378 & 0.5595 & 0.6954 \\
\hline Pipe velocities & $v_{17}$ & $v_{18}$ & $v_{19}$ & $v_{20}$ & $v_{21}$ & $v_{22}$ & $v_{23}$ & $v_{24}$ \\
\hline Values & 0.8994 & 0.5436 & 0.8672 & 0.9547 & 0.7783 & 0.7783 & 0.7702 & 0.6500 \\
\hline
\end{tabular}

\subsection{The accuracy of first iteration solution}

In fluid mechanics basics, the algebraic sum of the head losses around a loop must be zero which is not shown in tables 7 and 8 .

Table 7: The losses of each pipe for the first iteration

\begin{tabular}{|l|l|l|l|l|l|l|l|l|}
\hline No. losses & $h_{L 1}$ & $h_{L 2}$ & $h_{L 3}$ & $h_{L 4}$ & $h_{L 5}$ & $h_{L 6}$ & $h_{L 7}$ & $h_{L 8}$ \\
\hline Values & 1.0055 & 1.7795 & 3.2227 & 3.2227 & 3.1226 & 3.6390 & 0.6589 & 3.8234 \\
\hline No. losses & $h_{L 9}$ & $h_{L 10}$ & $h_{L 11}$ & $h_{L 12}$ & $h_{L 13}$ & $h_{L 14}$ & $h_{L 15}$ & $h_{L 16}$ \\
\hline Values & 2.9242 & 1.7850 & 7.3278 & 4.5572 & 4.6284 & 7.5305 & 1.8169 & 2.8668 \\
\hline No. losses & $h_{L 17}$ & $h_{L 18}$ & $h_{L 19}$ & $h_{L 20}$ & $h_{L 21}$ & $h_{L 22}$ & $h_{23}$ & $h_{L 24}$ \\
\hline Values & 3.6964 & 0.6398 & 3.6364 & 3.2845 & 2.5007 & 2.5007 & 1.3932 & 0.7747 \\
\hline
\end{tabular}

Table 8: The summation of losses in each loop for the first iteration

\begin{tabular}{|l|l|l|l|l|l|}
\hline Loop number & $F_{16}$ (loop1) & $F_{17}$ (loop2) & $F_{18}$ (loop3) & $F_{19}$ (loop4) & $F_{20}$ (loop5) \\
\hline Summation of head & 0.1622 & -1.6611 & 1.5378 & -0.8956 & -0.0138 \\
\hline Loop number & $F_{21}$ (loop6) & $F_{22}$ (loop7) & $F_{23}$ (loop8) & $F_{24}$ (loop9) & - \\
\hline Summation of head & 0.8592 & 0.1000 & 1.8255 & -0.0748 & - \\
\hline
\end{tabular}


4.8 The accuracy of last iteration solution

By using newton Raphson methods and using MATLAB code we got the sum of head loss around each loop is zero as shown in tables 9 and 10.

Table 9: The losses of each pipe for the last iteration

\begin{tabular}{|l|l|l|l|l|l|l|l|l|}
\hline No. losses & $h_{L 1}$ & $h_{L 2}$ & $h_{L 3}$ & $h_{L 4}$ & $h_{L 5}$ & $h_{L 6}$ & $h_{L 7}$ & $h_{L 8}$ \\
\hline Values & 1.0248 & 1.9004 & 2.8160 & 2.8160 & 3.9089 & 3.4130 & 0.6454 & 3.7925 \\
\hline No. losses & $h_{L 9}$ & $h_{L 10}$ & $h_{L 11}$ & $h_{L 12}$ & $h_{L 13}$ & $h_{L 14}$ & $h_{L 15}$ & $h_{L 16}$ \\
\hline Values & 2.3963 & 1.7232 & 6.6913 & 4.8680 & 5.0304 & 7.3063 & 1.5165 & 2.2339 \\
\hline No. losses & $h_{L 17}$ & $h_{L 18}$ & $h_{L 19}$ & $h_{L 20}$ & $h_{L 21}$ & $h_{L 22}$ & $h_{23}$ & $h_{L 24}$ \\
\hline Values & 3.5464 & 0.5970 & 3.3206 & 3.9505 & 2.7335 & 2.7335 & 1.6040 & 0.8228 \\
\hline
\end{tabular}

Table 10: The summation of losses in each loop for the last iteration

\begin{tabular}{|l|l|l|l|l|l|}
\hline Loop number & $F_{16}$ (loop1) & $F_{17}$ (loop2) & $F_{18}$ (loop3) & $F_{19}$ (loop4) & $F_{20}$ (loop5) \\
\hline Summation of head & 0 & 0 & 0 & 0 & 0 \\
\hline Loop number & $F_{21}$ (loop6) & $F_{22}$ (loop7) & $F_{23}$ (loop8) & $F_{24}$ (loop9) & - \\
\hline Summation of head & 0 & 0 & 0 & 0 & - \\
\hline
\end{tabular}

\section{The Solution By Using Hard Darcy}

The overall procedure for the looped network analysis can be summarized in the following steps:

1. Number all the node and pipe links, Also number the loops, for clarity, pipe numbers are circled and the loop numbers are put in square brackets.

2. Adopt a sign convention that a pipe discharge is positive if it flows from a lower node number the higher node number, otherwise negative.

3. Apply nodal continuity equation at all nodes to obtain pipe discharge .starting from nodes having two pipes with unknown discharge, assume an arbitrary discharge (say $0.1 \mathrm{~m}^{3} / \mathrm{s}$ ) in one of the pipes and apply continuityto obtain discharge in the other pipe. Repeat the procedure until all the pipe flows are known .if there exist more than two pipes having unknown discharges, assume arbitrary discharges in all the pipe except one and apply continuity equation to get discharge in the other pipe. The total number of primary loops in the network.

4. Assume friction factors $f_{i}=0.02$ in all pipes links and compute corresponding $K_{i}$

5. Assume loop pipe flow sign convention to apply loop discharge corrections; generally, clockwise flows positive and counterclockwise flows negative are considered.

6. Calculate $\Delta Q_{k}$ for the existing pipe flows and apply pipe corrections algebraically.

7. Apply the similar procedure in all the loops of a pipe network.

Repeat steps 6 and 7 until the discharge corrections in all the loops are relatively very small (Swamee\& Sharma,2008).

\subsection{The assumption}

The initial discharges should satisfy continuity equation at each node as table 11 (Moosavian\& Jaefarzadeh,2014). Also, the number of assumed discharge should be equaled to the number of loops which is nine.

Table 11: The assumed initial guesses for the first iteration

\begin{tabular}{|l|c|c|c|c|c|c|c|c|c|}
\hline Pipe discharge & $Q_{1}$ & $Q_{2}$ & $Q_{3}$ & $Q_{8}$ & $Q_{9}$ & $Q_{10}$ & $Q_{15}$ & $Q_{16}$ & $Q_{17}$ \\
\hline The assumed value & 0.10 & 0.03 & 0.02 & 0.02 & 0.02 & 0.01 & 0.02 & 0.02 & 0.02 \\
\hline
\end{tabular}

Then the rest of the discharge of the first iteration are listed in table 12.

Table 5.12: The discharge obtained from continuity equation

\begin{tabular}{|l|c|c|c|c|c|c|c|c|}
\hline Pipe discharge & $Q_{4}$ & $Q_{5}$ & $Q_{6}$ & $Q_{7}$ & $Q_{11}$ & $Q_{12}$ & $Q_{13}$ & $Q_{14}$ \\
\hline The assumed values & 0.02 & 0.03 & 0.07 & 0.05 & 0.01 & 0.04 & 0.07 & 0.03 \\
\hline Pipe discharge & $Q_{18}$ & $Q_{19}$ & $Q_{20}$ & $Q_{21}$ & $Q_{22}$ & $Q_{23}$ & $Q_{24}$ & - \\
\hline The assumed values & 0.03 & 0.04 & 0.07 & 0.01 & 0.01 & 0.08 & 0.12 & - \\
\hline
\end{tabular}




\subsection{The equation of Hard Darcy method}

* The discharge equation of each node

We know the summation of inflow and out flow at node should be equal zero, therefore:

$F_{1}=Q_{1}+Q_{7}-0.15$

$F_{2}=Q_{4}-Q_{3}$

$F_{3}=Q_{22}-Q_{21}$

$F_{4}=Q_{2}+Q_{6}-Q_{1}$

$F_{5}=Q_{3}+Q_{5}-Q_{2}$

$F_{6}=Q_{8}+Q_{14}-Q_{7}$

$F_{7}=Q_{15}+Q_{21}-Q_{14}$

$F_{8}=Q_{9}+Q_{13}-Q_{6}-Q_{8}$

$F_{10}=Q_{18}-Q_{17}-Q_{11}$

$F_{11}=Q_{10}+Q_{12}-Q_{5}-Q_{9}$

$F_{12}=Q_{23}-Q_{22}-Q_{20}$

$F_{13}=Q_{19}+Q_{17}-Q_{12}-Q_{16}$

$F_{14}=Q_{24}-Q_{19}-Q_{23}$

$F_{15}=Q_{11}-Q_{10}-Q_{4}$

* The loss equation

The algebraic sum of the head loss in a loop must be equal to to zero $\sum_{\text {loop }, k} k_{i} Q_{i}\left|Q_{i}\right|=0$ for all loops $k=1,2,3, \ldots \ldots, k_{L}$

Where $K_{i}=\frac{8 f_{i} L_{i}}{\pi^{2} g D_{i}^{5}}$

5.3 The first iteration of Hard Darcy method

Table 13 to table 21 show the calculation of the first iteration of each loop.

Where:

$$
\Delta Q_{k}=-\frac{\sum_{\text {loop }, k} K_{i} Q_{i}\left|Q_{i}\right|}{2 \sum_{\text {loop }, k} K_{i}\left|Q_{i}\right|}
$$

\begin{tabular}{|l|l|l|l|l|l|}
\hline Pipe & $\begin{array}{l}\text { Discharge } \\
\left(\mathrm{m}^{3} / \mathrm{s}\right)\end{array}$ & $\begin{array}{l}K \\
\left(\mathrm{~s}^{2} / \mathrm{m}^{5}\right)\end{array}$ & $\begin{array}{l}K Q|Q| \\
(\mathrm{m})\end{array}$ & $\begin{array}{l}2 K|Q| \\
\left(\mathrm{s} / \mathrm{m}^{2}\right)\end{array}$ & $\begin{array}{l}\text { Corrected Flow } \\
Q=Q+\Delta Q \\
\left(\mathrm{~m}^{3} / \mathrm{s}\right)\end{array}$ \\
\hline 1 & 0.1 & 129.1045 & 1.2910 & 25.8209 & 0.0747 \\
\hline 6 & 0.07 & 3098.5 & 15.1827 & 433.7910 & 0.0447 \\
\hline 7 & -0.05 & 96.8283 & -0.2421 & 9.6828 & -0.0753 \\
\hline Total & -0.02 & 3098.5 & -1.2394 & 123.9403 & -0.0453 \\
\hline$\Delta Q$ & & & 14.9923 & 593.2350 & \\
\hline
\end{tabular}

Table 13: loop 1 
Table 14: loop 2

\begin{tabular}{|l|l|l|l|l|l|}
\hline Pipe & $\begin{array}{l}\text { Discharge } \\
\left(\mathrm{m}^{3} / \mathrm{s}\right)\end{array}$ & $\begin{array}{l}K \\
\left(\mathrm{~s}^{2} / \mathrm{m}^{5}\right)\end{array}$ & $\begin{array}{l}K Q|Q| \\
(\mathrm{m})\end{array}$ & $\begin{array}{l}2 K|Q| \\
\left(\mathrm{s}_{\left.\mathrm{m}^{2}\right)}\right.\end{array}$ & $\begin{array}{c}\text { Corrected Flow } \\
Q=Q+\Delta Q \\
\left(\mathrm{~m}^{3} / \mathrm{s}\right)\end{array}$ \\
\hline 2 & 0.03 & 544.0452 & 0.4896 & 32.6427 & 0.0434 \\
\hline 5 & 0.03 & 3098.5 & 0.3099 & 61.9701 & 0.0234 \\
\hline 6 & -0.0447 & 3098.5 & -6.1988 & 277.1798 & -0.0313 \\
\hline 9 & -0.02 & 3098.5 & -1.2394 & 123.9403 & -0.0066 \\
\hline Total & & -6.6388 & 495.7330 & \\
\hline
\end{tabular}

Table 15: loop 3

\begin{tabular}{|l|l|l|l|l|l|}
\hline Pipe & $\begin{array}{l}\text { Discharge } \\
\left(\mathrm{m}^{3} / \mathrm{s}\right)\end{array}$ & $\begin{array}{l}K \\
\left(\mathrm{~s}^{2} / \mathrm{m}^{5}\right)\end{array}$ & $\begin{array}{l}\text { Corrected Flow } \\
(\mathrm{m})\end{array}$ & $\begin{array}{l}2 K|Q| \\
\left(\mathrm{s}_{\mathrm{m}}^{2}\right)\end{array}$ & $\begin{array}{l}Q=Q \\
\left(\mathrm{~m}^{3} / \mathrm{s}\right)\end{array}$ \\
\hline 3 & 0.02 & 4131.3 & 1.6525 & 165.2537 & 0.0176 \\
\hline 4 & 0.02 & 4131.3 & 1.6525 & 165.2537 & 0.0176 \\
\hline 5 & -0.0234 & 3098.5 & -1.6954 & 144.9593 & -0.0258 \\
\hline 10 & -0.01 & 3098.5 & -0.3099 & 61.9701 & -0.0124 \\
\hline Total & & & 1.2998 & 537.4369 & \\
\hline$\Delta Q$ & & -0.0024 & & \\
\hline
\end{tabular}

Table 16: loop 4

\begin{tabular}{|l|l|l|l|l|l|}
\hline Pipe & $\begin{array}{l}\text { Discharge } \\
\left(\mathrm{m}^{3} / \mathrm{s}\right)\end{array}$ & $\begin{array}{l}K \\
\left(\mathrm{~s}^{2} / \mathrm{m}^{5}\right)\end{array}$ & $\begin{array}{l}\text { K } \\
(\mathrm{m})\end{array}$ & $\begin{array}{l}2 K|Q| \\
\left(\mathrm{s}^{2} \mathrm{~m}^{2}\right)\end{array}$ & $\begin{array}{c}\text { Corrected Flow } \\
Q=Q+\Delta Q \\
\left(\mathrm{~m}^{3} / \mathrm{s}\right)\end{array}$ \\
\hline 10 & 0.0124 & 3098.5 & 0.4778 & 76.9577 & 0.0109 \\
\hline 11 & 0.01 & 3098.5 & 2.7887 & 185.9104 & 0.0285 \\
\hline 12 & -0.04 & 3098.5 & -1.2394 & 123.9403 & -0.0215 \\
\hline 17 & -0.02 & 3098.5 & -1.2394 & 123.9403 & -0.0215 \\
\hline Total & & 0.7877 & 510.7487 & \\
\hline$\Delta Q$ & & -0.0015 & & \\
\hline
\end{tabular}

Table 17 : loop 5

\begin{tabular}{|l|l|l|l|l|l|}
\hline Pipe & $\begin{array}{l}\text { Discharge } \\
\left(\mathrm{m}^{3} / \mathrm{s}\right)\end{array}$ & $\begin{array}{l}K \\
\left(\mathrm{~s}^{2} / \mathrm{m}^{5}\right)\end{array}$ & $\begin{array}{l}K Q|Q| \\
(\mathrm{m})\end{array}$ & $\begin{array}{l}2 K|Q| \\
\left(\mathrm{s}^{2} \mathrm{~m}^{2}\right)\end{array}$ & $\begin{array}{l}\text { Corrected Flow } \\
Q= \\
\left(\mathrm{m}^{3} / \mathrm{s}\right)\end{array}$ \\
\hline 9 & 0.0066 & 3098.5 & 0.1353 & 40.9511 & 0.0269 \\
\hline 12 & 0.0215 & 3098.5 & 1.4379 & 133.4976 & 0.0418 \\
\hline 13 & -0.07 & 3098.5 & -15.1827 & 433.7910 & -0.0497 \\
\hline 16 & -0.02 & 3098.5 & -1.2394 & 123.9403 & 0.00028 \\
\hline Total & & & -14.8489 & 732.1800 & \\
\hline
\end{tabular}

Table 18: loop 6

\begin{tabular}{|l|l|l|l|l|l|}
\hline Pipe & $\begin{array}{l}\text { Discharge } \\
\left(\mathrm{m}^{3} / \mathrm{s}\right)\end{array}$ & $\begin{array}{l}K \\
\left(\mathrm{~s}^{2} / \mathrm{m}^{5}\right)\end{array}$ & $\begin{array}{l}K Q|Q| \\
(\mathrm{m})\end{array}$ & $\begin{array}{l}2 K|Q| \\
\left(\mathrm{s} / \mathrm{m}^{2}\right)\end{array}$ & $\begin{array}{c}\text { Corrected Flow } \\
Q=Q \Delta Q \\
\left(\mathrm{~m}^{3} / \mathrm{s}\right)\end{array}$ \\
\hline 8 & 0.0453 & 3098.5 & 6.3506 & 280.5514 & 0.0342 \\
\hline 13 & 0.0497 & 3098.5 & 7.6596 & 308.1134 & 0.0386 \\
\hline 14 & -0.03 & 3098.5 & -2.7887 & 185.9104 & -0.0411 \\
\hline 15 & -0.02 & 3098.5 & -1.2394 & 123.9403 & -0.0311 \\
\hline Total & & & 9.9822 & 898.5156 & \\
\hline$\Delta Q$ & & -0.0111 & & \\
\hline
\end{tabular}


Table 19: loop7

\begin{tabular}{|l|l|l|l|l|l|}
\hline Pipe & $\begin{array}{l}\text { Discharge } \\
\left(\mathrm{m}^{3} / \mathrm{s}\right)\end{array}$ & $\begin{array}{l}K \\
\left(\mathrm{~s}^{2} / \mathrm{m}^{5}\right)\end{array}$ & $\begin{array}{l}K Q|Q| \\
(\mathrm{m})\end{array}$ & $\begin{array}{l}2 K|Q| \\
\left(\mathrm{s}^{2} \mathrm{~m}^{2}\right)\end{array}$ & $\begin{array}{l}\text { Corrected Flow } \\
Q=Q+\Delta Q \\
\left(\mathrm{~m}^{3} / \mathrm{s}\right)\end{array}$ \\
\hline 15 & 0.0311 & 3098.5 & 2.9988 & 192.7867 & 0.0077 \\
\hline 20 & 0.07 & 3098.5 & 15.1827 & 433.7910 & 0.0466 \\
\hline 22 & -0.01 & 3098.5 & -0.3099 & 61.9701 & -0.0334 \\
\hline Total & -0.01 & 3098.5 & -0.3099 & 61.9701 & -0.0334 \\
\hline$\Delta Q$ & & & 17.5617 & 750.5180 & \\
& & -0.0234 & & \\
\end{tabular}

Table 20: loop 8

\begin{tabular}{|l|l|l|l|l|l|}
\hline Pipe & $\begin{array}{l}\text { Discharge } \\
\left(\mathrm{m}^{3} / \mathrm{s}\right)\end{array}$ & $\begin{array}{l}K \\
\left(\mathrm{~s}^{2} / \mathrm{m}^{5}\right)\end{array}$ & $\begin{array}{l}K Q|Q| \\
(\mathrm{m})\end{array}$ & $\begin{array}{l}2 K|Q| \\
\left(\mathrm{s}^{2} \mathrm{~m}^{2}\right)\end{array}$ & $\begin{array}{c}\text { Corrected Flow } \\
Q= \\
\left(\mathrm{m}^{3} / \mathrm{s}\right)\end{array}$ \\
\hline 16 & 0.00028 & 3098.5 & 0.000243 & 1.7373 & 0.0172 \\
\hline 19 & 0.04 & 3098.5 & 1.2394 & 123.9403 & 0.0369 \\
\hline 20 & -0.0334 & 3098.5 & -6.7287 & 288.7840 & -0.0297 \\
\hline 23 & -0.08 & 408.0339 & -2.6114 & 65.2854 & -0.0631 \\
\hline Total & & & -8.1005 & 479.7470 & \\
\hline$\Delta Q$ & & 0.0169 & & \\
\hline
\end{tabular}

Table 21: loop 9

\begin{tabular}{|l|l|l|l|l|l|}
\hline Pipe & $\begin{array}{l}\text { Discharge } \\
\left(\mathrm{m}^{3} / \mathrm{s}\right)\end{array}$ & $\begin{array}{l}K \\
\left(\mathrm{~s}^{2} / \mathrm{m}^{5}\right)\end{array}$ & $\begin{array}{l}K Q|Q| \\
(\mathrm{m})\end{array}$ & $\begin{array}{l}2 K|Q| \\
\left(\mathrm{s}^{2}\right)^{2}\end{array}$ & $\begin{array}{c}\text { Corrected Flow } \\
Q Q \\
\left(\mathrm{~m}^{3} / \mathrm{s}\right)\end{array}$ \\
\hline 17 & 0.0215 & 3098.5 & 1.4379 & 133.4976 & 0.0305 \\
\hline 18 & 0.03 & 96.8283 & 0.2421 & 9.6828 & 0.0590 \\
\hline 19 & -0.0369 & 3098.5 & -4.2155 & 228.5766 & -0.0279 \\
\hline 24 & -0.12 & 96.8283 & -0.9683 & 19.3657 & -0.0910 \\
\hline Total & & & -3.5038 & 391.1228 & \\
\hline$\Delta Q$ & & 0.0090 & & \\
\hline
\end{tabular}

5.4 The pipe discharges for the first iteration

The discharges of the first iteration are shown in table 22 by (MATLAB code).

Table 22: The pipe discharge of the first iteration

\begin{tabular}{|l|l|l|l|l|l|l|l|l|}
\hline Pipe discharge & $Q_{1}$ & $Q_{2}$ & $Q_{3}$ & $Q_{4}$ & $Q_{5}$ & $Q_{6}$ & $Q_{7}$ & $Q_{8}$ \\
\hline Values & 0.0747 & 0.0367 & 0.0231 & 0.0231 & 0.0336 & 0.0380 & 0.0753 & 0.0329 \\
\hline Pipe discharge & $Q_{9}$ & $Q_{10}$ & $Q_{11}$ & $Q_{12}$ & $Q_{13}$ & $Q_{14}$ & $Q_{15}$ & $Q_{16}$ \\
\hline Values & 0.0289 & 0.0262 & 0.0193 & 0.0463 & 0.0420 & 0.0424 & 0.0089 & 0.0112 \\
\hline Pipe discharge & $Q_{17}$ & $Q_{18}$ & $Q_{19}$ & $Q_{20}$ & $Q_{21}$ & $Q_{22}$ & $Q_{23}$ & $Q_{24}$ \\
\hline Values & 0.0308 & 0.0501 & 0.0267 & 0.0397 & 0.0335 & 0.0335 & 0.0732 & 0.0999 \\
\hline
\end{tabular}

\subsection{The pipe discharges and velocities of the last iteration}

The correct discharges and velocities can be got after many number of iteration (MATLAB code by using Hard Darcy method), showed in tables 23 and 24.

Table 23: The pipe discharges for the last iteration

\begin{tabular}{|l|l|l|l|l|l|l|l|l|}
\hline Pipe discharge & $Q_{1}$ & $Q_{2}$ & $Q_{3}$ & $Q_{4}$ & $Q_{5}$ & $Q_{6}$ & $Q_{7}$ & $Q_{8}$ \\
\hline Values & 0.0786 & 0.0507 & 0.0213 & 0.0213 & 0.0294 & 0.0279 & 0.0714 & 0.0296 \\
\hline Pipe discharge & $Q_{9}$ & $Q_{10}$ & $Q_{11}$ & $Q_{12}$ & $Q_{13}$ & $Q_{14}$ & $Q_{15}$ & $Q_{16}$ \\
\hline Values & 0.0232 & 0.0187 & 0.0401 & 0.0339 & 0.0343 & 0.0418 & 0.0174 & 0.0226 \\
\hline Pipe discharge & $Q_{17}$ & $Q_{18}$ & $Q_{19}$ & $Q_{20}$ & $Q_{21}$ & $Q_{22}$ & $Q_{23}$ & $Q_{24}$ \\
\hline Values & 0.0285 & 0.0686 & 0.0274 & 0.0297 & 0.0243 & 0.0243 & 0.0540 & 0.0814 \\
\hline
\end{tabular}


In addition, by apply the equation [4] $Q=v A_{C}$ we get the following velocities:

Table 24: The pipe velocities for the last iteration

\begin{tabular}{|l|l|l|l|l|l|l|l|l|}
\hline Velocities & $v_{1}$ & $v_{2}$ & $v_{3}$ & $v_{4}$ & $v_{5}$ & $v_{6}$ & $v_{7}$ & $v_{8}$ \\
\hline Values & 0.6257 & 0.7179 & 0.6796 & 0.6796 & 0.9357 & 0.8875 & 0.5680 & 0.9420 \\
\hline Velocities & $v_{9}$ & $v_{10}$ & $v_{11}$ & $v_{12}$ & $v_{13}$ & $v_{14}$ & $v_{15}$ & $v_{16}$ \\
\hline Values & 0.7389 & 0.5966 & 1.2762 & 1.0779 & 1.0907 & 1.3299 & 0.5554 & 0.7199 \\
\hline Velocities & $v_{17}$ & $v_{18}$ & $v_{19}$ & $v_{20}$ & $v_{21}$ & $v_{22}$ & $v_{23}$ & $v_{24}$ \\
\hline Values & 0.9071 & 0.5458 & 0.8729 & 0.9970 & 0.7745 & 0.7745 & 0.7638 & 0.6478 \\
\hline
\end{tabular}

\subsection{The accuracy of first iteration solution}

The solution that showed above only for the first iteration, which is not correct. The next test, shows that $\Delta Q$ are not equal to zero which is not correct as shown in table 25

Table 25: The correction factor in each loop of the first iteration

\begin{tabular}{|l|l|l|l|l|l|l|l|l|l|l|}
\hline No. loop & 1 & 2 & 3 & 4 & 5 & 6 & 7 & 8 & 9 \\
\hline$\Delta Q$ & -0.0253 & 0.0067 & 0.0031 & 0.0093 & 0.0156 & -0.0124 & -0.0235 & 0.0068 & 0.0201 \\
\hline
\end{tabular}

5.7 The accuracy of last iteration solution

Hard Darcy method by using MATLAB code was run to get the next results as a proof of the accuracy of the solution of the discharges as shown in table 26

Table 26: The correction factor in each loop of the last iteration

\begin{tabular}{|l|l|l|l|l|l|l|l|l|l|}
\hline No. loop & 1 & 2 & 3 & 4 & 5 & 6 & 7 & 8 & 9 \\
\hline$\Delta Q$ & 0 & 0 & 0 & 0 & 0 & 0 & 0 & 0 & 0 \\
\hline
\end{tabular}

\section{Flow Rate Comparison}

The differences between the discharges obtained by Newton Raphson and Hard Darcy method are approximately zero as shown in fig (2)

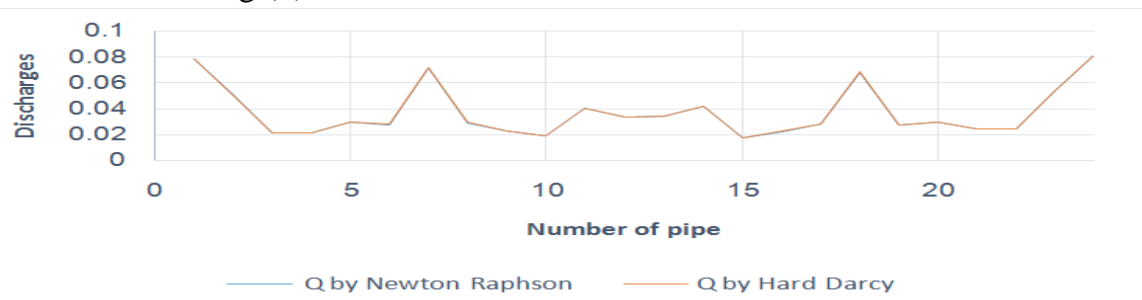

Fig 2 : Flow rates obtained by Newton Raphson and Hard Darcy methods with number pi

\section{The Number Of Iteration With The Summation Of Head Losses In Each Loop For Newton Raphson Method}

The correct flow rates by Newton Raphson method were got after 3 iteration as shown fig (3).

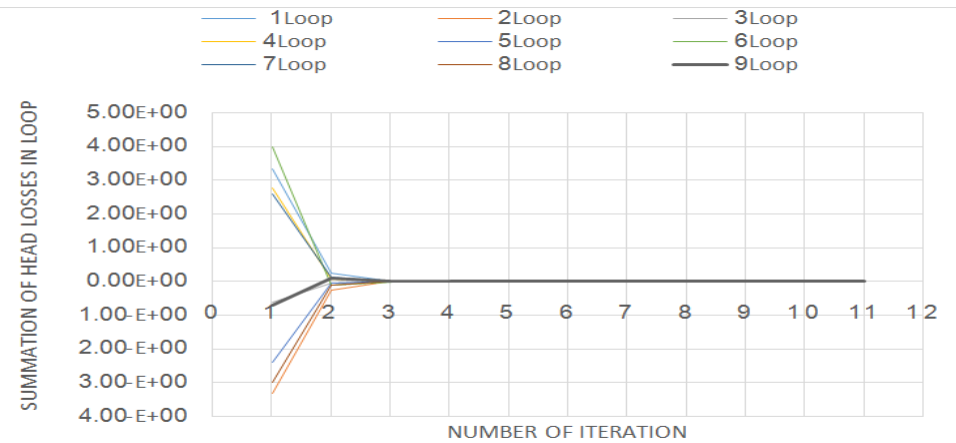

Fig 3: The relationship between the numbers of iteration with the summation of head losses equations in each loop for Newton Raphson method 


\section{The Number Of Iteration With The Summation Of Head Losses In Each Loop For Hard Darcy Method}

The correct flow rates by Hard Darcy method were got after 20 iteration as shown fig (4).

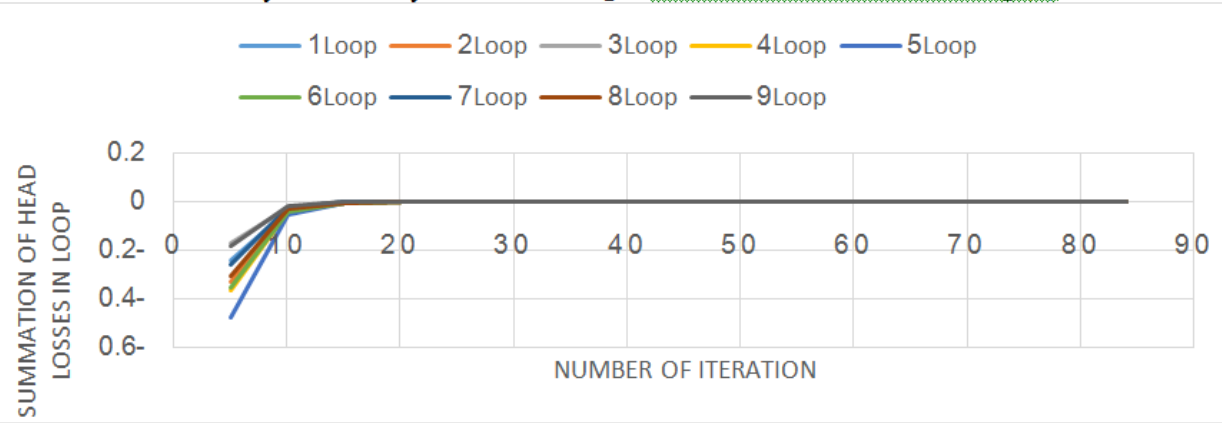

Fig 4: The relationship between the numbers of iteration with the summation of head losses equations in each loop for Hard Darcy method

\section{Comparison Between The Summation Of Head Losses Equations By Newton Raphson And Hard Darcy}

The next table shows the summation of the head loss equation in each loop that must be approximately zero, which can be seen that newton Raphson is faster than hard Darcy to converge to the solution.

Table 27: Thesummation head losses equations by Newton Raphson and Hard Darcy methods

\begin{tabular}{|l|l|l|}
\hline Loop number & $\begin{array}{l}\text { The summation of head losses (Newton } \\
\text { Raphson) after 11 iteration. (m) }\end{array}$ & $\begin{array}{l}\text { The summation of head losses (Hard Darcy) } \\
\text { after 84 iteration.(m) }\end{array}$ \\
\hline 1 & $-5.5511 \mathrm{e}-016$ & $1.1102 \mathrm{e}-016$ \\
\hline 2 & 0 & $-4.4409 \mathrm{e}-016$ \\
\hline 3 & $-8.8818 \mathrm{e}-016$ & 0 \\
\hline 4 & $-1.7764 \mathrm{e}-015$ & $-8.8818 \mathrm{e}-016$ \\
\hline 5 & $8.8818 \mathrm{e}-016$ & $-1.3323 \mathrm{e}-015$ \\
\hline 6 & 0 & $-8.8818 \mathrm{e}-016$ \\
\hline 7 & 0 & $-4.4409 \mathrm{e}-016$ \\
\hline 8 & 0 & $-8.8818 \mathrm{e}-016$ \\
\hline 9 & $-1.8127 \mathrm{e}-006$ & $-4.4409 \mathrm{e}-016$ \\
\hline
\end{tabular}

\section{Conclusion}

A nonlinear systems network were simulated by Newton Raphson and Hard Darcy methods using MATLAB software. The nonlinearity is showed in the square power of the discharge in head losses equations. The discharges resulted of each pipe were found the same in each method. Also, the final solution was validated by using the basic of fluid mechanics which that the summation of losses inside a loop must be equal to zero. Thus numerically, in Newton Raphson, which summation has a high accuracy and approximately zero compared to Hard Darcy method. Also, the solution in Newton Raphson method can be got at less number of iterations (faster) compared to Hard Darcy method. In addition, initial guesses (the assumption) is more complicated in Hard Darcy because the value of each discharge must satisfy the continuity equations which need more calculations. However, the initial guesses can be chosen randomly in Newton Raphson method without satisfying the continuity equations.

\section{References}

[1]. Hund-Der Yeh\& Yu-Chang Lin.pipe network system analysis using simulated annealing. journal of water supplyResearch and technology- AQUA;aug2008Vol 57(5) 317:327.

[2]. Abdulhamid El IdrisSaad. Design of Nonlinear Piping Water System Network by Using Newton Raphson Method. IOSR Journal of Mechanical and Civil Engineering (IOSR-JMCE); Mar-Apr 2016Vol 13 (2) 33:39.

[3]. Yunus A. Cengel, John M. Cimbala . fluid mechanics fundamental and application , Higher Education;2006.

[4]. Bruce R. Munson, Donald F. Young, Theodore H. Okiishi, Wade W. Huebsch, fundamental of fluid mechanics, Sixth Edition, John Wiley\&Sons.INC;2009.

[5]. M. Tabesh, T. T. Tanyimboh, R. Burrows. Head-driven simulation of water supply networks. J SID;2001.

[6]. N. Moosavian*,M. R. Jaefarzadeh. Hydraulic Analysis of water supply networks using a modified hard Darcy method. International Journal of Engineering;September2014Vol 27 (9) 1331:1338.

[7]. I.A.Oke, Reliabililty and statistical assessment of methods for pipe network analysis, environmental engineering science, December 2007 Vol 24 (10) 1481:1490.

[8]. Prabhata K. Swamee, Ashok K. Sharma. Designof water supplu pipe network, John Wiley \& Sons, Inc; 2008 